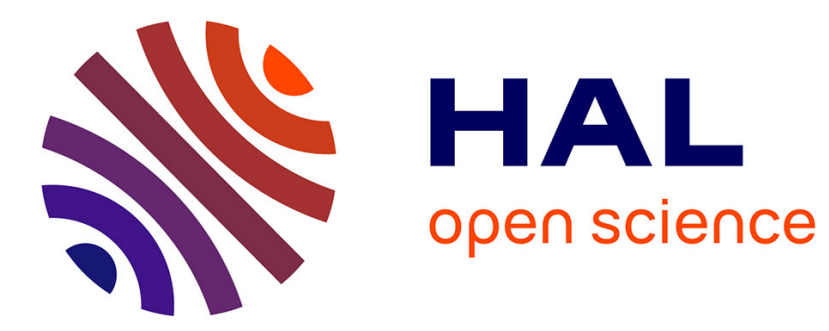

\title{
Le recrutement et la fidélisation des bénévoles retraités par les associations
}

Andréa Gourmelen, Samuel Guillemot, Hélène Privat, Bertrand Urien, Marine Le Gall-Ely

\section{- To cite this version:}

Andréa Gourmelen, Samuel Guillemot, Hélène Privat, Bertrand Urien, Marine Le Gall-Ely. Le recrutement et la fidélisation des bénévoles retraités par les associations. Décisions Marketing, 2014, 73, pp.105-118. 10.7193/DM.073.109.122 . hal-01205965

\section{HAL Id: hal-01205965 \\ https://hal.science/hal-01205965}

Submitted on 28 Sep 2015

HAL is a multi-disciplinary open access archive for the deposit and dissemination of scientific research documents, whether they are published or not. The documents may come from teaching and research institutions in France or abroad, or from public or private research centers.
L'archive ouverte pluridisciplinaire HAL, est destinée au dépôt et à la diffusion de documents scientifiques de niveau recherche, publiés ou non, émanant des établissements d'enseignement et de recherche français ou étrangers, des laboratoires publics ou privés. 


\section{Le recrutement et la fidélisation des bénévoles retraités par les associations}

\section{Version auteur}

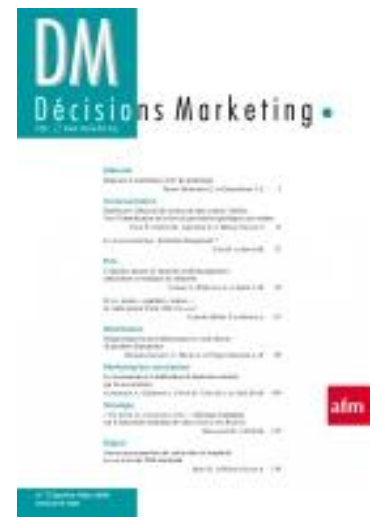

\section{L'article de la revue est disponible ici :}

http://www.editions-ems.fr/revues/decisions-marketing/articlerevue/1103-lerecrutement-et-la-fid\%C3\%A9lisation-de-b\%C3\%A9n\%C3\%A9volesretrait\%C3\%A9s-par-les-associations.html

Pour citer cet article

Gourmelen Andréa, Guillemot Samuel, Privat Hélène, Urien Bertrand et Le GallEly Marine (2014), Le recrutement et la fidélisation des bénévoles retraités par les associations, Décisions Marketing, 73, 105-118

\section{Résumé}

En raison du temps libre engendré par l'arrêt de l'activité professionnelle, les retraités deviennent une cible très convoitée par les associations à la recherche de bénévoles. Cependant, ils sont souvent considérés comme un segment homogène, d'où des difficultés de recrutement et de fidélisation par les associations. Pour y remédier, cet article propose une typologie de bénévoles retraités sur la base de leurs motivations et de caractéristiques psychosociales liées au vieillissement. Cinq profils se dégagent : les hédonistes, les coupables, les affectifs, les soucieux de postérité, les ambitieux. Reste aux associations à cibler un ou plusieurs profils selon la fonction à pourvoir et à adapter leurs campagnes de communication en conséquence.

Mots-clés : Bénévoles, retraités, typologie, motivations, générativité, sagesse 


\section{Le recrutement et la fidélisation des bénévoles retraités par les associations}

\section{Introduction}

«Devenez bénévole! », «Bénévole, pourquoi pas moi ?», "Qui d'autre que vous?». Ces slogans se développent dans les campagnes de communications associatives. Pour cause, en France, gérer la motivation des bénévoles est la préoccupation principale des dirigeants d'associations, et ce, devant la situation financière de leur organisation (étude Recherches \& Solidarités, janvier 2012). Sachant que $80 \%$ des associations n'ont pas de salarié (Birambeau et Larceneux, 2008), le recrutement et la fidélisation des bénévoles sont des enjeux nécessaires à la survie de notre paysage associatif. Le bénévolat est généralement considéré comme un don de temps (Prouteau et Wolff, 2004), à toute personne extérieure à son foyer (Archambault et Prouteau, 2009). On comprend alors pourquoi les retraités sont des cibles privilégiées pour pallier le manque de bénévoles. En effet, en raison du temps libéré par la cessation d'activité professionnelle, ces derniers peuvent potentiellement s'engager sur l'année, assurer des permanences en semaine et mettre leur expérience au profit des jeunes bénévoles (Cerphi, 2010). Cependant, pourquoi donner son temps à une association plutôt qu'à une autre ?

En pratique, le recrutement et la fidélisation des bénévoles retraités s'avèrent difficiles. Le recours à des slogans insistant sur le besoin en bénévoles se généralise et au final, les campagnes de communication des associations tendent à se ressembler. De surcroît, les associations ont l'habitude de considérer les retraités comme des « réservoirs » de temps en les affectant aux tâches les plus urgentes, sans se soucier de leurs souhaits (Van Ingen et Dekker, 2011). Or, ce segment est constitué de personnes hétérogènes en termes d'âge, de situation familiale, de vécu du vieillissement, d'attentes et de besoins (Guiot et Urien, 2012).

La littérature marketing qui se focalise davantage sur le don d'objets (Bergadaà, 2006) et d'argent (Sargeant, Ford et West, 2006) n'offre que peu de solutions concernant le don de temps. C'est pourquoi, notre objectif est de proposer un outil aux responsables associatifs afin qu'ils tiennent compte de la diversité des bénévoles retraités et de leurs motivations; de manière à mieux les recruter et les fidéliser. Pour y parvenir, nous allons présenter les différences interindividuelles concernant les motivations au 
bénévolat d'une part et les modifications psychosociales liées à l'avancée en âge issues du cadre théorique d'Erikson d'autre part. Nous élaborerons ensuite une typologie des bénévoles retraités en cinq profils sur la base de ces différences interindividuelles. Enfin, nous discuterons des possibilités d'utilisation de cette typologie par les associations, comme la création de campagnes de communication plus ciblées. A la clé, un recrutement plus efficace et une diminution du turn-over.

\section{Pourquoi donner son temps ? Les motivations au bénévolat des retraités}

Les recherches relatives au don de temps en tant que comportement à part entière sont davantage développées en psychologie (Clary, Snyder et Stukas, 1996 ; Chantal et Vallerand, 2000) et en sociologie (Sue et Peter, 2011). En marketing, quelques rares travaux relatifs au bénévolat l'étudient de manière concomitante au don d'argent (Fennis, Janssen et Vohs, 2009).

De plus, toutes disciplines confondues, rares sont les travaux qui se focalisent sur les retraités (Tang, Morrow-Howell et Hong, 2009), malgré l'intérêt porté à cette cible par les associations. Pourtant, outre certaines activités informelles pour lesquelles ils sont souvent sollicités, comme la garde des petits-enfants, les bénévoles retraités ont la réputation de donner davantage d'heures aux associations que les bénévoles plus jeunes (Cerphi, 2010 ; Prouteau et Wolff, 2007). Or, à ce sujet, France Bénévolat met en garde sur son site internet les responsables associatifs contre les clichés qui perdurent: l'engagement des retraités dans le bénévolat serait la conséquence d'une réflexion, de motivations, et non la résultante automatique d'une augmentation du temps disponible. Une synthèse de la littérature montre effectivement que les motivations au bénévolat se retrouvent à tout âge ; seul leur ordre d'importance varierait (Clary, Snyder et Stukas, 1996 ; Sue et Peter, 2011). Elles peuvent être classées selon le bénéficiaire perçu de l'action et leur degré d'autodétermination (tableau 1). 


\section{Tableau 1}

Classification des motivations au bénévolat adaptée de Chantal et Vallerand (2000).

\begin{tabular}{|c|c|c|}
\hline Motivations & Altruistes & Egoïstes \\
\hline $\begin{array}{c}\text { Auto- } \\
\text { déterminées }\end{array}$ & $\begin{array}{l}\text { Origine : SOI } \\
\text { Bénéficiaire : AUTRUI } \\
\text { Exemple : par choix face aux } \\
\text { problèmes de la société. }\end{array}$ & $\begin{array}{l}\text { Origine : SOI } \\
\text { Bénéficiaire : SOI } \\
\text { Exemples : pour découvrir de } \\
\text { nouvelles activités ; pour faire des } \\
\text { rencontres agréables. }\end{array}$ \\
\hline $\begin{array}{l}\text { Non auto- } \\
\text { déterminées }\end{array}$ & $\begin{array}{c}\text { Origine : AUTRUI } \\
\text { Bénéficiaire : AUTRUI } \\
\text { Exemple : par obligation morale. }\end{array}$ & $\begin{array}{l}\text { Origine : AUTRUI } \\
\text { Bénéficiaire : SOI } \\
\text { Exemples : pour acquérir de } \\
\text { l'expérience professionnelle ; pour } \\
\text { être reconnu socialement. }\end{array}$ \\
\hline
\end{tabular}

\section{Le bénéficiaire perçu de l'action : soi ou autrui}

La classification des motivations selon leur nature altruiste (bénéficiaire : autrui) ou égoïste (bénéficiaire: soi) apparaît comme une évidence dans de nombreux travaux relatifs au bénévolat (Bussell et Forbes, 2002 ; Sue et Peter, 2011). Cependant, notons que ces deux catégories de motivations peuvent coexister chez un même individu. Par exemple, une personne peut faire du bénévolat à la fois pour aider son prochain (motivation altruiste) et pour être reconnue socialement (motivation égoïste). Ainsi, l'altruisme «pur» est difficilement envisageable. Cette classification, parfois controversée (Prouteau et Wolff, 2004), s'avère alors plus pertinente lorsqu'elle est complétée par un second critère, celui du degré d'autodétermination (Chantal et Vallerand, 2000), qui fait référence à l'origine de la motivation.

\section{L'origine de la motivation : soi ou autrui}

La dichotomie opposant les motivations autodéterminées (origine: soi) et non autodéterminées (origine : autrui) fait référence au degré de liberté ressenti par un individu lors d'une prise de décision (Deci et Ryan, 1985). Si une personne perçoit son 
engagement bénévole comme un choix librement consenti, elle est motivée de manière dite «autodéterminée ». Tel est le cas lorsqu'un individu s'investit dans le bénévolat parce qu'il juge que c'est en adéquation avec sa personnalité, ou encore pour faire des rencontres (Chantal et Vallerand, 2000). Au contraire, un individu qui perçoit sa décision comme influencée par des «forces » externes, est motivé de manière «non autodéterminée ». Tel est le cas lorsqu'il pratique le bénévolat parce qu'il ressent une obligation morale, une pression extérieure, ou encore parce qu'il espère une certaine reconnaissance (Chantal et Vallerand, 2000).

Ainsi, les motivations au bénévolat peuvent se classer en quatre grandes familles, mais toutes ne sont pas pour autant présentes chez chaque bénévole. Comment alors mobiliser les connaissances relatives aux effets de l'avancée en âge sur la personnalité pour expliquer le développement ou non de certaines motivations?

\section{L’influence du vieillissement sur les motivations au bénévolat}

La théorie psychosociale du développement de la personnalité d'Erikson (encadré 1) constituera notre cadre d'analyse. Erikson est, en effet, l'un des rares théoriciens de la personnalité à examiner le vieillissement comme une étape du développement de la personnalité, au même titre que la petite enfance, l'enfance, l'adolescence, et la vie adulte. Dans ce sens, il met en évidence deux préoccupations qui vont forger le comportement dans la dernière partie de sa vie : le désir de participer au cycle des générations et celui de comprendre la vie pour accepter sa mort. Il faut bien garder à l'esprit que ces caractéristiques peuvent apparaître plus tôt dans le cycle de vie mais qu'elles sont particulièrement saillantes chez les personnes vieillissantes qui ont plus de temps et plus de moyens pour les cultiver.

Encadré 1 : la théorie du développement de la personnalité d'Erikson (1959)

Erikson considère la vie comme une succession de huit «crises existentielles » qui mettent en jeu un pôle positif et un pôle négatif. L'enjeu développemental consiste à trouver un équilibre entre ces deux extrêmes. Dans le scénario idéal d'Erikson, lorsqu'une personne a résolu la crise identitaire associée à l'adolescence ( $5^{\text {ème }}$ étape), puis qu'elle a établi une relation d'intimité ( $6^{\text {ème }}$ étape), elle est alors prête à employer 
son énergie pour le bien-être des générations futures ( $7^{\mathrm{e} m e}$ étape). Cette inclinaison générative diminuerait ensuite avec l'âge pour laisser place à la recherche dite de sagesse $\left(8^{\text {ème }}\right.$ étape). Cette théorie de développement «par étape » est parfois controversée car elle ne prend pas suffisamment en compte de variables culturelles et individuelles, comme si tous les individus de par le monde grandissaient et vieillissaient de la même manière. Néanmoins, les chercheurs reconnaissent qu'Erikson a introduit des concepts clés (Pietikainen et Ihanus, 2003).

\section{Participer au cycle des générations : la générativité}

Selon Erikson, les individus vieillissants qui commencent à raisonner en « temps qu'il reste à vivre » deviennent préoccupés par leur contribution à la société (désir d'être utile aux autres) et le souvenir qu'ils laisseront (désir d'immortalité symbolique). Aussi, les deux facettes (altruiste et égoïste) qui caractérisent les motivations au bénévolat semblent se retrouver dans la générativité. En effet, une personne générative agira de manière narcissique (dimension égoïste), en faisant en sorte que ses actions reflètent son identité - tout en veillant à ce qu'elles soient profitables à la génération suivante (dimension altruiste) (McAdams, De St Aubin et Logan, 1993).

La générativité est en réalité un concept multidimensionnel où chaque composante influencerait plus particulièrement certains types de motivations au bénévolat :

\section{- La générativité postérité : ne pas être oublié}

La dimension postérité de l'intérêt génératif correspond à l'intérêt d'une personne à ne pas être oubliée par la génération suivante. Cette préoccupation à laisser une empreinte durable sur les autres peut engendrer des motivations au bénévolat de type égoïste non autodéterminé (bénéficiaire: soi ; origine: autrui car l'individu espère une reconnaissance). Ainsi, la générativité postérité peut engendrer par exemple la prise de responsabilités dans une association dans le but d'être reconnu socialement.

\section{- La générativité transmission : passer le relais à la jeune génération}

La dimension transmission fait référence à l'intérêt d'une personne à transmettre des éléments (connaissances, etc.) aux générations suivantes. Elle souligne un trait de caractère altruiste, pouvant se manifester, par exemple, chez un retraité qui va accepter 
de s'occuper de ses petits-enfants de manière à rendre service à sa famille. Elle est donc susceptible d'entraîner des motivations autodéterminées altruistes d'une part (le bénévolat est le moyen choisi pour passer le relais à la jeune génération), et égoïstes d'autre part (le bénévolat permet d'entretenir un lien social avec les générations suivantes).

\section{- La générativité communion : agir pour la société}

La dimension communion souligne l'intérêt d'un individu à contribuer à la vie de la collectivité, de la société. Cette dimension souligne un engagement envers la communauté duquel peut découler une obligation morale et donc engendrer des motivations au bénévolat non autodéterminées altruistes, de type : «je pratique le bénévolat parce que les autres comptent sur moi ». Le bénévolat n'est donc pas dans ce cas une activité librement choisie, mais un devoir vis-à-vis d'autrui, de la société.

\section{Comprendre la vie pour accepter sa mort : la sagesse}

La $8^{\text {ème }}$ et dernière étape d'Erikson concerne la recherche d'intégrité personnelle. Cette tâche est à rapprocher du concept de relecture de vie (Butler, 1963). Toute personne confrontée de manière directe ou indirecte à sa propre mortalité se plonge dans une introspection de manière à résoudre les conflits de sa vie passée et ainsi appréhender l'échéance ultime de manière plus sereine. Cette introspection peut engendrer un bilan négatif, dans le cas où l'individu constaterait une différence entre ce qu'il espérait devenir et ce qu'il est devenu. Au contraire, un bilan positif permet de comprendre et d'accepter sa vie. Il permet ainsi de cultiver et développer la sagesse ; dernière étape de la théorie d'Erikson.

Or, la sagesse est également un concept multidimensionnel (dimensions cognitive, réflexive et affective - Ardelt ; 2003, 2004). C'est la composante affective de la sagesse qui semble la plus propice à induire des motivations au bénévolat. En effet, celle-ci concerne la capacité d'un individu à être compréhensif et compatissant envers les autres et représente ainsi une propension à aller vers l'autre et à donner. La sagesse affective peut par conséquent expliquer des motivations de type autodéterminées altruistes du type : «je fais du bénévolat, car je trouve naturel d'aider ceux qui ont besoin d'aide ». 
A partir de ces constats, notre objectif est maintenant de dresser une typologie de bénévoles retraités sur la base de leurs motivations et de discriminer leurs profils par les dimensions de la générativité et de la sagesse que nous venons de mettre en évidence.

\section{Une typologie de bénévoles retraités en cinq profils}

La typologie a été élaborée sur la base d'une étude quantitative par questionnaire (encadré 2) et les profils obtenus sont analysés ci-dessous :

\section{Encadré 2 : éléments méthodologiques}

\section{L'échantillon}

Pour appartenir à l'échantillon il fallait être retraité et bénévole. Les 202 individus interrogés âgés de 50 à 88 ans $^{1}$ (âge moyen de 66,41 ans) pratiquent une activité bénévole essentiellement dans des associations à dominante sociale (Restos du cœur, Secours Populaire, Unicef, etc.), et dans une moindre mesure dans des associations de type «loisirs-culture » (bibliothèque, musées, clubs de pétanque etc.). Ces deux catégories d'associations correspondent à celles privilégiées, en France, par les bénévoles âgés (Prouteau et Wolff, 2007).

\section{Instruments de mesure}

Nous avons utilisé des échelles de mesure existantes dans la littérature interdisciplinaire pour appréhender les construits à mesurer. Nous avons vérifié la fiabilité et la stabilité des instruments de mesure à l'aide d'analyses factorielles (annexe A1) :

$>$ Une version simplifiée de l'échelle de motivation envers l'action bénévole (Chantal et Vallerand, 2000) composée de 12 items se répartissant sur 4 facteurs représentant les 4 types de motivations au bénévolat.

3 dimensions de la Loyola Generative Scale (McAdams et De St Aubin, 1992 ; Urien et Kilbourne, 2011) composée de 8 items pour mesurer les dimensions postérité (GP), transmission (GT) et communion (GC) de l'intérêt génératif.

La sagesse affective a été mesurée en utilisant 3 items de la Three Dimensional Wisdom Scale (Ardelt, 2003).

\footnotetext{
${ }^{1}$ Notons que les personnes dont l'âge est inférieur à 60 ans sont bien retraitées. Il s'agit essentiellement de retraités de la Marine Nationale.
} 


\section{Analyses typologiques}

Nous avons mené l'analyse sur les scores factoriels issus des différentes dimensions mesurées. La typologie a été bâtie en deux temps :

- Une classification avec une méthode hiérarchique (méthode ascendante avec l'algorithme de Ward) nous a permis d'envisager des structures à 4, 5 voire 6 groupes.

- La structure en 5 groupes a été retenue avec une méthode non hiérarchique (algorithme des K-means). En effet, celle-ci présente une portée opérationnelle: les individus qui composent les segments sont suffisamment nombreux pour que l'on puisse en déduire des actions managériales à mener (plus de 10\% de l'effectif total).

\section{Résultats}

Afin d'interpréter au mieux cette typologie, nous nous sommes intéressés aux centres de classe des construits mesurés issus des analyses factorielles. Pour appréhender les écarts entre les groupes, nous avons utilisé les tests de significativité (tableau 2).

\section{Tableau 2.}

\section{Typologie en 5 profils}

\begin{tabular}{|c|c|c|c|c|c|c|}
\hline & 1 & 2 & 3 & 4 & 5 \\
\hline & & $\begin{array}{c}\text { Les } \\
\text { bénévoles } \\
\text { hédonistes } \\
\end{array}$ & $\begin{array}{c}\text { Les } \\
\text { bénévoles } \\
\text { coupables } \\
\end{array}$ & $\begin{array}{c}\text { Les } \\
\text { bénévoles } \\
\text { affectifs }\end{array}$ & $\begin{array}{c}\begin{array}{c}\text { Les bénévoles } \\
\text { soucieux de } \\
\text { postérité }\end{array} \\
\end{array}$ & $\begin{array}{c}\text { Les } \\
\text { bénévoles } \\
\text { ambitieux } \\
\end{array}$ \\
\hline $\begin{array}{l}\text { Variables } \\
\text { «actives » }\end{array}$ & $\begin{array}{l}\text { Motivations au } \\
\text { bénévolat } \\
\text { Autodét. Altruistes } \\
\text { Autodét. Egoïstes } \\
\text { Non Autodét. Altruistes } \\
\text { Non Autodét. Egoïstes } \\
\end{array}$ & $\begin{array}{c}-1,74_{a} \\
0,42_{a} \\
-0,33_{a} \\
-0,43_{a} \\
\end{array}$ & $\begin{array}{c}0,00_{\mathrm{b}} \\
-1,31_{\mathrm{b}} \\
1,00_{\mathrm{b}} \\
-0,62_{\mathrm{a}} \\
\end{array}$ & $\begin{array}{c}0,34_{\mathrm{b}} \\
0,55_{\mathrm{a}} \\
-0,01_{\mathrm{a}} \\
-0,52_{\mathrm{a}} \\
\end{array}$ & $\begin{array}{l}0,27_{\mathrm{b}} \\
0,49_{\mathrm{a}} \\
0,54_{\mathrm{b}} \\
1,40_{\mathrm{b}} \\
\end{array}$ & $\begin{array}{c}0,02_{\mathrm{b}} \\
-0,95_{\mathrm{b}} \\
-1,00_{\mathrm{c}} \\
0,36_{\mathrm{c}} \\
\end{array}$ \\
\hline \multirow{4}{*}{$\begin{array}{c}\text { Variables } \\
\text { «muettes » }\end{array}$} & \begin{tabular}{|l} 
Générativité \\
Postérité \\
Transmission \\
Communion \\
\end{tabular} & $\begin{array}{l}-0,43_{a} \\
-0,19_{a} \\
-0,73_{a} \\
\end{array}$ & $\begin{array}{c}-0,19_{a} \\
-0,18_{a} \\
0,26_{b} \\
\end{array}$ & $\begin{array}{c}-0,04_{a} \\
-0,08_{a} \\
0,05_{b} \\
\end{array}$ & $\begin{array}{l}0,62_{\mathrm{b}} \\
0,27_{\mathrm{a}} \\
0,16_{\mathrm{b}} \\
\end{array}$ & $\begin{array}{c}-0,03_{\mathrm{a}, \mathrm{b}} \\
-0,06_{\mathrm{a}} \\
-0,06_{\mathrm{a}, \mathrm{b}} \\
\end{array}$ \\
\hline & $\begin{array}{l}\text { Sagesse } \\
\text { Affective }\end{array}$ & $-0,27_{\mathrm{a}, \mathrm{b}}$ & $0,01_{a, b}$ & $0,23_{\mathrm{a}}$ & $-0,02_{a, b}$ & $-0,40_{b}$ \\
\hline & Age & $66_{a}$ & $63_{\mathrm{a}}$ & $67_{\mathrm{a}}$ & $68_{a}$ & $66_{a}$ \\
\hline & $\begin{array}{c}\text { Effectifs } \\
\%\end{array}$ & $\begin{array}{c}19 \\
(\mathbf{1 1 \%} \%)\end{array}$ & $\begin{array}{c}23 \\
(13 \%)\end{array}$ & $\begin{array}{c}69 \\
(\mathbf{3 9 \%})\end{array}$ & $\begin{array}{c}33 \\
(\mathbf{1 9 \%})\end{array}$ & $\begin{array}{c}34 \\
(\mathbf{1 9 \%})\end{array}$ \\
\hline
\end{tabular}

Légende : Les valeurs des mêmes lignes ne partageant pas le même indice $(a, b, c)$ diffèrent significativement à $p<$ 0.05 dans le test bilatéral d'égalité pour moyennes de colonne. Les tests sont ajustés à l'aide de la correction Bonferroni. 


\section{Profil 1 : Les hédonistes}

Les individus qui composent ce profil (11\%) sont caractérisés par la présence de motivations uniquement autodéterminées et égoïstes (origine : soi, bénéficiaire : soi). Le bénévolat représente pour eux une activité divertissante, leur permettant de découvrir de nouvelles opportunités. Dans ce sens, leur activité bénévole s'apparente davantage à un loisir et à une source d'épanouissement personnel qu'à un acte altruiste. Cette manière de percevoir le bénévolat peut s'expliquer par un intérêt génératif relativement faible. Ainsi, ces individus donneraient leur temps sans en avoir l'impression, tant la découverte et l'amusement tiennent une place prépondérante dans leur activité bénévole.

\section{Profil 2 : Les coupables}

Par opposition au profil précédent, les individus composant ce second groupe (13\%) se caractérisent par de faibles motivations égoïstes et par de très fortes motivations altruistes non autodéterminées (origine : autrui, bénéficiaire : autrui). Ces personnes font alors du bénévolat par principe, par devoir moral, et font preuve d'une véritable abnégation. Pour eux, donner du temps pour des personnes qui en ont besoin représente un acte contraint, un devoir de rendre à la société ce qu'elle leur a donné. En effet, certaines personnes se sentent vraiment coupables d'avoir eu une belle vie ou d'avoir été, d'une manière ou d'une autre, privilégiées dans la vie. Ainsi, le bénévolat est ici, un moyen de rendre à la société ce qu'elle leur a donné. Ce profil de bénévoles est marqué par une prédominance de la composante communion de la générativité. Ainsi, ce sont des personnes qui se sentent impliquées dans le fonctionnement de la société.

\section{Profil 3 : Les affectifs}

Les bénévoles affectifs sont les plus nombreux (39\%) et se démarquent par le caractère autodéterminé de leurs motivations (origine: soi ; bénéficiaire : soi et autrui). Le bénévolat est pour eux une activité librement choisie, qui leur permet de se faire plaisir tout en aidant les autres. Cela leur permet d'être en accord avec leur personnalité et leur procure de la joie; d'où la coexistence de motivations altruistes et égoïstes. Ce sont des bénévoles dévoués aux autres, qui retirent des bénéfices affectifs des liens tissés. Il n'est 
pas surprenant de constater que la composante affective de la sagesse est développée chez ces personnes. Ainsi, ces bénévoles se caractériseraient par beaucoup d'empathie et un goût prononcé pour les relations humaines.

\section{Profil 4 : Les soucieux de postérité}

Ce groupe de bénévoles (19\%) se caractérise par la présence de plusieurs motivations, toutes globalement élevées. Parmi elles, se détachent particulièrement les motivations non autodéterminées égoïstes (origine : autrui ; bénéficiaire : soi). Pour ces personnes, le bénévolat est un moyen de satisfaire un besoin de reconnaissance à travers des contacts sociaux. Ces bénévoles sont par ailleurs caractérisés par un intérêt génératif lié à la postérité davantage développé que chez les autres groupes. Ces personnes souhaitent donc ne pas être oubliées par la génération suivante. Cela nous laisse à penser que ces personnes, préoccupées par le souvenir qu'elles laisseront, valorisent le bénévolat comme un moyen de laisser leur empreinte.

\section{Profil 5 : Les ambitieux}

Ce profil (19\%) s'apparente au précédent au sens où les motivations non autodéterminées égoïstes (origine : autrui ; bénéficiaire : soi) sont les plus développées ; à un degré moindre cependant. La différence réside dans la faible intensité des autres motivations. Ainsi, les intentions des ambitieux semblent claires : utiliser le bénévolat pour parvenir à leurs fins (recherche de reconnaissance sociale, de prestige, voire de pouvoir), sans se soucier des liens humains pouvant être noués ou des codes sociaux et moraux. Ce profil est caractérisé par le plus faible niveau de sagesse affective : les relations humaines seraient considérées comme un moyen d'atteindre plus de reconnaissance.

Cette typologie montre la diversité des bénévoles retraités, ainsi que la cohérence entre motivations et caractéristiques psychosociales du vieillissement. Comment peut-elle contribuer à réduire les inquiétudes des responsables associatifs à propos du recrutement et de la fidélisation des bénévoles? 


\section{Voies d'utilisation de la typologie pour le recrutement et la fidélisation des bénévoles}

Cette dernière partie a pour vocation à présenter des exemples d'utilisation de la typologie de manière à atteindre nos deux objectifs initiaux : aider à recruter et fidéliser les bénévoles retraités. Ainsi, nous répondrons à deux questions : comment utiliser la typologie de bénévoles retraités pour créer des campagnes de recrutement originales et plus efficaces ? Puis, comment optimiser l'affectation des tâches en fonction du profil des bénévoles?

\section{Un outil pour se démarquer de campagnes qui se ressemblent}

Pour répondre à la première question, nous avons choisi de différencier les réponses en fonction de l'objectif de la campagne de communication associative. Nous illustrerons nos propos par des exemples de campagnes récentes correspondant à différents objectifs.

\section{- Recruter divers profils : de « pourquoi pas moi ? » à « pourquoi moi ?»}

De nombreuses associations souhaitent accroître le nombre de leurs bénévoles de manière importante. Pour cela, elles mettent en avant la diversité des profils de ceux qui sont d'ores et déjà engagés à leurs côtés, essentiellement en termes d'âge et de profession. Ce type de communication a vocation à s'adresser à tous, sous-entendant qu'il existe toujours une mission, une activité bénévole qui nous correspond au sein de l'association, quel que soit notre profil. Cependant, la banalisation de ce type de campagne entraîne la généralisation de slogans de type : «rejoignez-nous », «pourquoi pas moi ? », «qui d'autre que vous? ». Si cela semble pertinent pour promouvoir le bénévolat en général, ce type de communication n'évoque pas l'intérêt de s'engager comme bénévole dans une organisation plutôt qu'une autre.

De plus, les retraités pour lesquels le bénévolat est une activité valorisante dans la société actuelle (profil 5: «les ambitieux») ou future (profil 4: «les soucieux de postérité ») peuvent craindre la banalisation et la dévalorisation de leurs activités, sous prétexte que «tout le monde peut devenir bénévole». A cet égard, l'exemple comparé 
de l'Eglise catholique et de l'Unicef est illustratif du manque de personnalisation des messages (encadré 3).

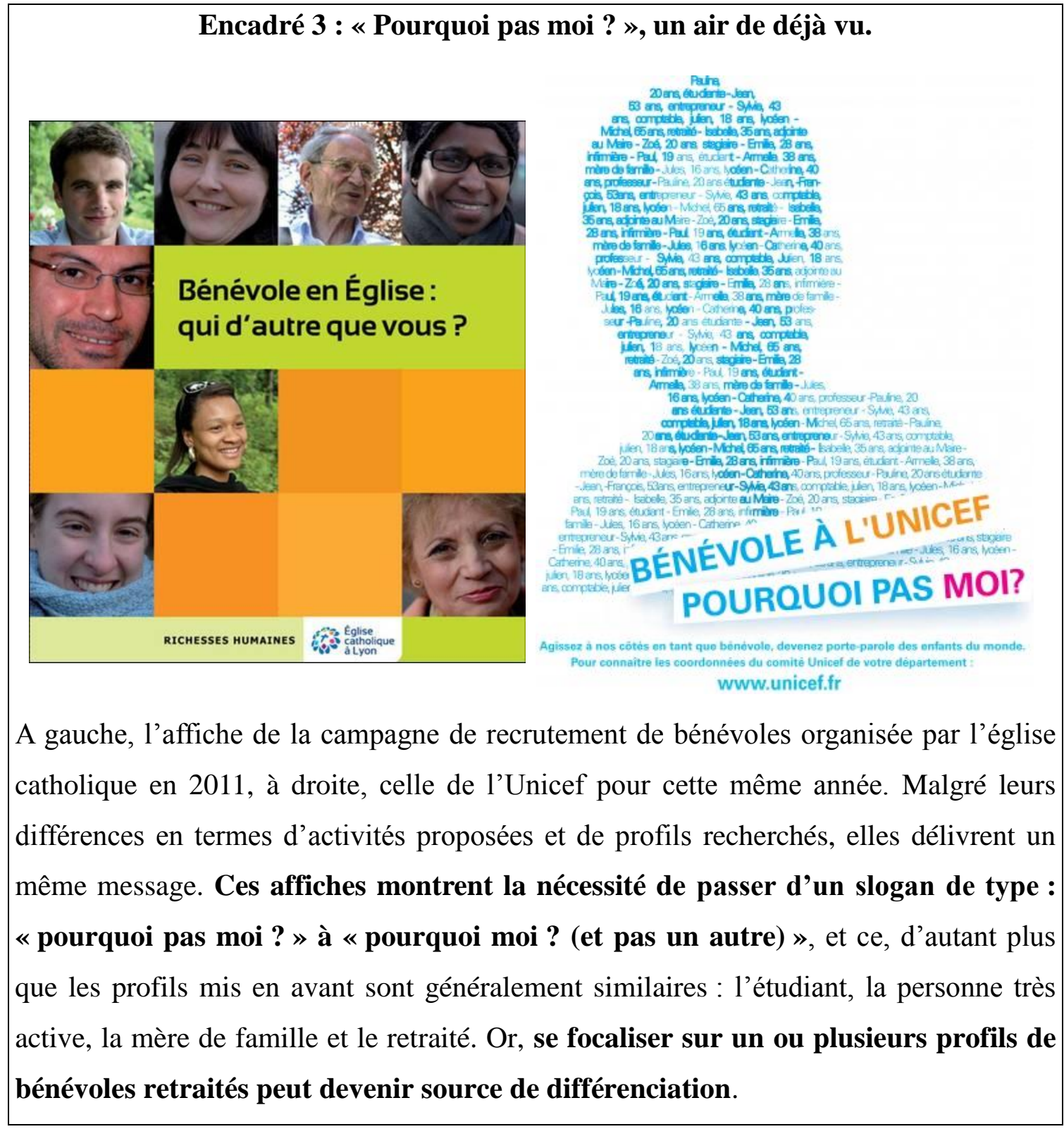

Utiliser la typologie de bénévoles retraités à des fins de ciblage des campagnes pourrait remédier à ce problème. En effet, une piste d'amélioration de ce type de campagne est de personnaliser le message en fonction du profil recherché. Par exemple, dans les spots publicitaires de l'Unicef, le retraité prononce la même phrase que l'étudiante et que la chef d'entreprise : "Pour venir en aide aux enfants, moi, j'ai choisi d'être bénévole », qui s'apparente à une motivation altruiste autodéterminée. Cependant, notre typologie laisse penser que ce type de motivation n'est pas présent de manière isolée chez les 
retraités. Il se couple à des motivations de même origine, mais de nature égoïste chez les bénévoles «affectifs» (profil 3). Evoquer uniquement une motivation altruiste autodéterminée revient à prendre un risque : celui d'être trop général, et par conséquent qu'aucun retraité ne se sente véritablement concerné par cette campagne. Mettre en valeur des motivations autodéterminées égoïstes en plus, c'est cibler un profil de retraité particulier doté d'une grande sagesse affective, qualité indéniable pour les missions humanitaires. Un slogan mettant en avant les deux types de motivations autodéterminées pourrait être : "Pour venir en aide aux enfants et construire une vraie relation d'amitié avec eux, moi, j'ai choisi d'être bénévole ».

- Recruter des retraités : de « nous recherchons » à « nous vous proposons » Certaines associations mettent en œuvre des campagnes de communication spécifiquement destinées à recruter des bénévoles retraités ou seniors. Ce type de campagne s'avère nécessaire pour certaines tâches impliquant une régularité de l'engagement, ou au contraire, un don de temps ponctuel pendant toute une semaine. Une particularité de ces campagnes est leur présentation, qui détaille les qualités que doivent posséder les candidats au bénévolat, telles des annonces d'emploi. Ce type d'accroche, de type : "nous recherchons... » montre le sérieux de la mission bénévole ; du moins la présence d'une réflexion sur les qualités recherchées pour la mener à bien. Dans le cas de fonctions de bureau à pourvoir (président, trésorier, secrétaire), cette ressemblance avec une offre d'emploi peut être un moyen d'attirer les ambitieux (profil 5), en leur donnant l'opportunité d'une nouvelle carrière et d'un certain pouvoir inhérent à la fonction. Cependant, dans les faits, les qualités évoquées restent bien souvent générales, telles que l'expérience, la motivation, l'envie de s'engager (encadré 4).

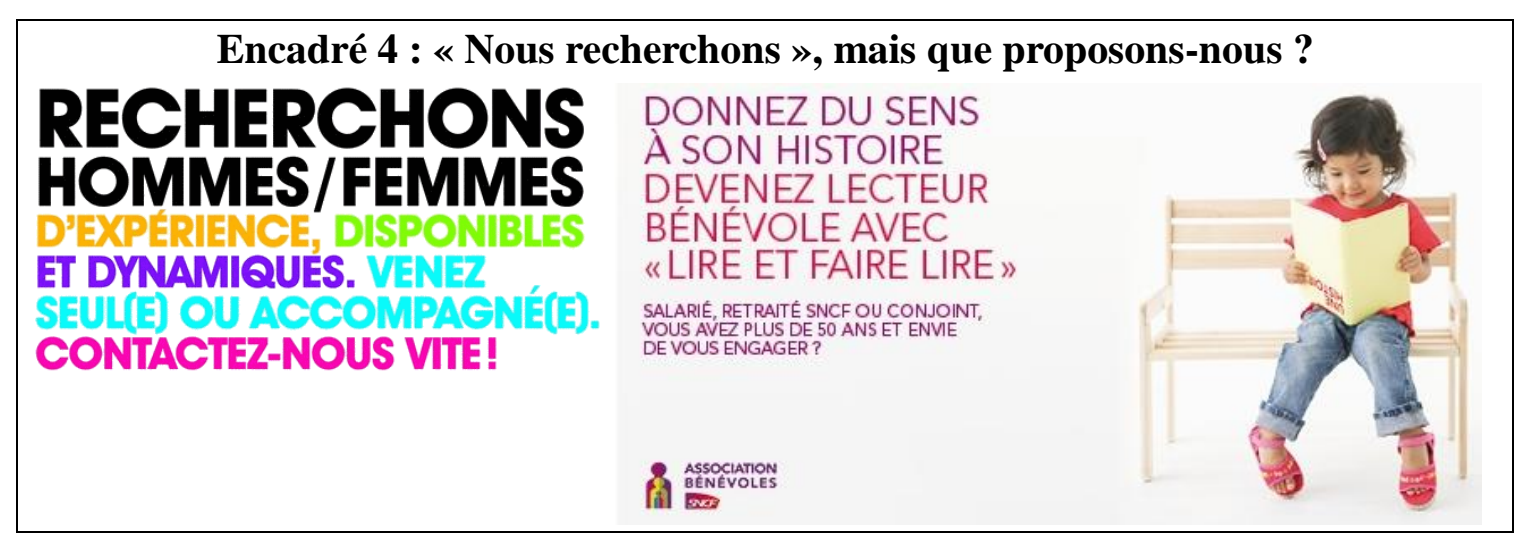


Ci-dessus, deux appels à bénévoles âgés ou retraités : à gauche pour le Téléthon, à droite pour l'association «Lire et faire lire », dans le cadre d'un partenariat avec la fondation SNCF. Malgré des activités différentes, le message de ces associations est similaire: «si vous correspondez à ce que nous cherchons, venez vite, nous avons besoin de vous». Cependant, les qualités recherchées sont presque identiques: des bénévoles ayant un certain vécu (âge ou expérience) et motivés (notion de dynamisme ou d'envie de s'engager).

Une piste d'amélioration concernant ce type de message est de donner cette envie de s'engager tant recherchée en communiquant sur les activités proposées, avec des mots en adéquation avec le profil de bénévole recherché pour se différencier. A titre d'exemple, «Lire et faire lire », pourrait exploiter la phrase «donnez du sens à son histoire » en y ajoutant la notion de générativité postérité. Un argument de type «vous resterez gravé dans sa mémoire » aura une résonnance auprès des «soucieux de postérité » (profil 4). Quant à l'appel pour le Téléthon, il pourra être complété par des mots tels que « groupe », « ambiance conviviale », «leçon de vie », qui font référence à la générativité communion caractérisant les bénévoles « coupables » (profil 2).

Pour se différencier, il conviendrait de communiquer sur ce que l'on propose (au lieu de ce que l'on recherche), en employant des termes correspondant à la personnalité des bénévoles recherchés (liés à la sagesse ou la générativité). Cela permettrait de donner cette envie de s'engager pour l'association, d'impulser cette motivation tant recherchée et d'élargir la cible de ce type de communication à divers profils de retraités.

\section{- Recruter et fidéliser : des remerciements avec modération}

Enfin, certaines campagnes de communication ont un double objectif: recruter de nouveaux bénévoles tout en fidélisant ceux déjà présents. La stratégie est alors de remercier publiquement les bénévoles, pour inciter les non-bénévoles à s'engager. Les slogans de type : "merci aux bénévoles », «bravo pour vos actions », se multiplient. Ces témoignages de reconnaissance, souvent évoqués comme indispensables envers les retraités (Tang, Morrow-Howell et Hong, 2009), semblent à manipuler avec précaution (encadré 5). Les « ambitieux » (profil 5) et les « soucieux de postérité » (profil 4) seront 
très sensibles à ces gestes et arguments de reconnaissance témoignés par les associations. Cependant, pour attirer et fidéliser d'autres profils, valoriser les bénévoles n'est pas la solution. Les «affectifs » (profil 3) et les «hédonistes » (profil 1) sont animés principalement par des motivations autodéterminées (origine: soi). Etre valorisé, mis en avant peut entrer en décalage avec leurs motivations et leur personnalité.

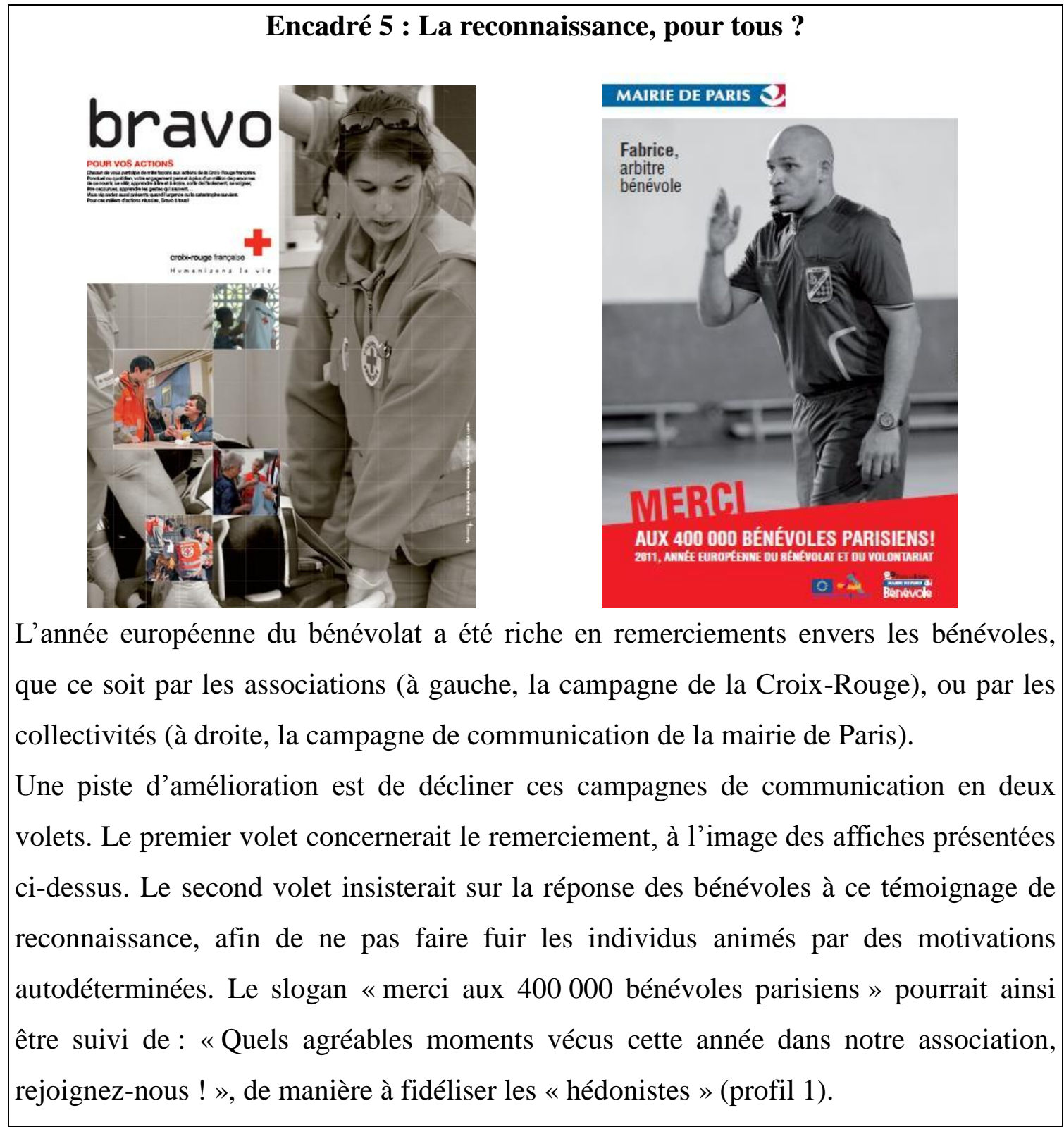

Ainsi, les campagnes de communication relatives au bénévolat sont susceptibles d'attirer, en l'état, une minorité de bénévoles retraités. La typologie en cinq profils 
permet d'améliorer le ciblage opéré par les associations afin d'attirer et fidéliser davantage de bénévoles retraités.

\section{Des fonctions pour chaque profil de bénévole retraité}

Si la communication est un élément important pour maintenir un effectif suffisant de bénévoles, les responsables associatifs doivent également veiller à ce que leurs promesses n'entrent pas en contradiction avec les missions proposées aux bénévoles. A défaut, ils augmentent le risque de «démission» de nombreux bénévoles à tout moment, étant donné la liberté inhérente à la notion de bénévolat. La communication doit ainsi être adaptée aux postes à pourvoir dans l'association. S'interroger sur le profil de retraité le plus adapté à chaque activité, c'est ainsi mettre en place une politique de fidélisation des bénévoles propre à chaque association, et encourager les comportements de bénévolat durables. Par exemple, pour une mission ponctuelle liée à l'organisation d'un événement, l'association peut mettre en avant la notion d'amusement, de loisir, pour attirer des retraités de type « hédonistes ». Inversement, elle ne peut pas insister sur ces éléments lorsqu'elle cherche à renouveler son bureau. Ce serait tromper les bénévoles hédonistes de les attirer via la notion de loisir, pour les affecter à des tâches telles que la comptabilité ou la trésorerie. Ainsi, concernant ce type de fonction, mieux vaut cibler les «ambitieux ». Le tableau 3 présente, au regard des caractéristiques des profils de bénévoles retraités, les fonctions correspondant à chacun d'eux, les actions à réaliser, ainsi que les arguments de communication susceptibles de les attirer.

\section{Tableau 3}

Répondre aux attentes de chaque profil : fonctions, actions, communication

\begin{tabular}{|c|c|c|c|}
\hline Profils & $\begin{array}{l}\text { Fonctions } \\
\text { adaptées }\end{array}$ & $\begin{array}{c}\text { Exemples d'actions à } \\
\text { proposer }\end{array}$ & $\begin{array}{l}\text { Arguments de } \\
\text { communication }\end{array}$ \\
\hline $\begin{array}{c}\text { 1. Les } \\
\text { hédonistes }\end{array}$ & $\begin{array}{l}\text { Animateur, } \\
\text { Guide, } \\
\text { Chargé de } \\
\text { communication, } \\
\text { Chargé } \\
\text { d'événementiel. }\end{array}$ & $\begin{array}{l}\text { - Organisation } \\
\text { d'événement festif, sportif, } \\
\text { culturel ; } \\
\text { - Graphisme, décoration; } \\
\text { - Visites de musées. }\end{array}$ & $\begin{array}{l}\text { Mise en avant du loisir, de } \\
\text { l'amusement. } \\
\text { Exemples : «Organisons } \\
\text { ensemble une grande } \\
\text { fête», «Enrichissement } \\
\text { culturel »... }\end{array}$ \\
\hline
\end{tabular}




\begin{tabular}{|c|c|c|c|}
\hline $\begin{array}{c}\text { 2. Les } \\
\text { coupables }\end{array}$ & $\begin{array}{l}\text { Chargé } \\
\text { d'accueil, } \\
\text { Ecoutant, } \\
\text { Assistant, } \\
\text { Formateur, } \\
\text { Educateur. }\end{array}$ & $\begin{array}{l}\text { - Ecoute de personnes en } \\
\text { difficulté ; } \\
\text { - Assistance juridique; } \\
\text { - Actions de formation, } \\
\text { d'insertion par la culture } \\
\text { ou le sport. }\end{array}$ & $\begin{array}{l}\text { Mise en avant du devoir } \\
\text { moral, de la notion de } \\
\text { justice sociale. } \\
\text { Exemples: "Changer les } \\
\text { choses», "Réduire les } \\
\text { inégalités», "Le droit à } \\
\text { une seconde chance »... }\end{array}$ \\
\hline $\begin{array}{c}\text { 3. Les } \\
\text { affectifs }\end{array}$ & $\begin{array}{l}\text { Parrain, } \\
\text { Intervenant } \\
\text { professionnel, } \\
\text { Enseignant. }\end{array}$ & $\begin{array}{l}\text { - Action humanitaire à } \\
\text { l'étranger (missions de } \\
\text { construction, } \\
\text { d'éducation...); } \\
\text { - Parrainage de proximité } \\
\text { (grands-parents d'accueil). }\end{array}$ & $\begin{array}{l}\text { Mise en avant du lien } \\
\text { humain, de l'aspect } \\
\text { relationnel, de la } \\
\text { réciprocité. } \\
\text { Exemples : «Je reçois plus } \\
\text { que je donne ; «Une } \\
\text { deuxième famille »... }\end{array}$ \\
\hline $\begin{array}{c}\text { 4. Les } \\
\text { soucieux } \\
\text { de } \\
\text { postérité }\end{array}$ & $\begin{array}{l}\text { Fonctions } \\
\text { caractère } \\
\text { officiel : } \\
\text { dirigeant, } \\
\text { président, } \\
\text { responsable. }\end{array}$ & $\begin{array}{l}\text { - Représentation de } \\
\text { l'association au forum des } \\
\text { associations de quartier; } \\
\text { - Discours, animation lors } \\
\text { de galas de charité, de } \\
\text { cérémonies, d'événements } \\
\text { sportifs. }\end{array}$ & $\begin{array}{l}\text { Mise en avant de la } \\
\text { reconnaissance post- } \\
\text { mortem, de la trace laissée } \\
\text { aux générations futures. } \\
\text { Exemples: «les enfants } \\
\text { vous remercient»; } \\
\text { «marquer les esprits», } \\
\text { "gravé dans la } \\
\text { mémoire»... }\end{array}$ \\
\hline $\begin{array}{c}\text { 5. Les } \\
\text { ambitieux }\end{array}$ & $\begin{array}{l}\text { Fonctions du } \\
\text { siège : président, } \\
\text { dirigeant, } \\
\text { trésorier, } \\
\text { comptable, } \\
\text { secrétaire. }\end{array}$ & $\begin{array}{l}\text { - Gestion de partenariats } \\
\text { avec les entreprises; } \\
\text { - Inaugurations; } \\
\text { - Comptabilité, trésorerie; } \\
\text { - Suivi de dossiers, de } \\
\text { demandes de subventions. }\end{array}$ & $\begin{array}{l}\text { Mise en avant de la } \\
\text { reconnaissance de ses } \\
\text { pairs, assimilation du } \\
\text { bénévolat à un travail, } \\
\text { notion de leadership. } \\
\text { Exemples: «Exercez vos } \\
\text { compétences», «Une } \\
\text { nouvelle carrière »... }\end{array}$ \\
\hline
\end{tabular}




\section{Conclusion}

Cette recherche a permis de mettre en lumière la diversité des bénévoles retraités via l'établissement d'une typologie en cinq profils, et de montrer l'utilité managériale de cette distinction. Notre typologie est ainsi de nature à aider les responsables associatifs à ne plus considérer leurs bénévoles âgés comme un ensemble homogène en se focalisant uniquement sur leur temps disponible. De plus, notre analyse montre que les variables liées au vieillissement ont un pouvoir discriminant plus important que des variables sociodémographiques telles que l'âge chronologique. Notons que d'autres variables caractéristiques du vieillissement subjectif, telles que l'âge subjectif (Guiot, 2006), pourraient être étudiées afin d'enrichir la typologie.

Considérer la diversité de ces bénévoles permet de répondre aux interrogations des responsables associatifs concernant le recrutement et la fidélisation des bénévoles. La réalisation de campagnes ciblant un ou plusieurs profils de retraités, en se focalisant sur leurs caractéristiques psychosociales et leurs motivations, permettra aux associations de se différencier. Par la suite, analyser les réactions des retraités aux messages des associations à travers des expérimentations pourrait constituer une voie de recherche future pour adapter plus facilement le contenu des messages selon le profil recherché. Certains profils peuvent effectivement être plus sensibles aux messages faisant écho à leurs motivations, d'autres à des références implicites à la générativité ou à la sagesse, par exemple.

Enfin, adapter les missions bénévoles en fonction du profil du retraité (pouvant être cerné à travers ses motivations) pourra constituer une action de fidélisation efficace pour faire face à la concurrence d'autres associations.

Notons pour terminer une précaution d'emploi à destination des dirigeants d'associations qui souhaiteraient utiliser cette typologie. Celle-ci n'est valable que sur des retraités. Les profils découlent en effet des dernières étapes de vie identifiées par Erikson (1959). Il convient donc de trouver d'autres arguments de communication lorsqu'il s'agit de «rajeunir » son équipe de bénévoles. Dans ce sens, une piste de recherche serait d'envisager le pouvoir prédictif d'autres variables de personnalité liées à des stades moins avancés de la théorie d'Erikson. 


\section{Bibliographie}

Archambault E. et Prouteau L. (2009), Mesurer le bénévolat pour en améliorer la connaissance et satisfaire à une recommandation internationale. RECMA - Revue internationale de l'économie sociale, 314, 84-104.

Ardelt M. (2003), Empirical assessment of a three-dimensional wisdom scale, Research on Aging, 25, 3, 275-324.

Ardelt M. (2004), Wisdom as expert knowledge system: a critical review of a contemporary operationalization of an ancient concept, Human Development, 47, 5, 257-285.

Bergadaà M. (2006), Le don d'objets : dimensions centrales et profils de donneurs aux œuvres de bienfaisance, Recherche et Applications en Marketing, 21, 1, 19-39.

Birambeau P. et Larceneux, F. (2008), Comment recruter, intégrer et fidéliser les bénévoles ? in Gallopel-Morvan K., Birambeau, P., Rieunier S. et Larceneux F. (eds), Marketing et communication des associations, Dunod, 13-44.

Bussell H. et Forbes D. (2002), Understanding the volunteer market: the what, where, who and why of volunteering, International Journal of Nonprofit and Voluntary Sector Marketing, 7, 3, 244-257.

Butler R.N. (1963), The life review: an interpretation of reminiscence in the age, Psychiatry, 26, 65-75.

Centre d'Etude et de Recherche sur la Philanthropie (Cerphi) (2010), Les associations et les seniors bénévoles : nouvelles relations et nouvelles pratiques.

Chantal Y. et Vallerand R. J. (2000), Construction et validation de l'échelle de motivation envers l'action bénévole (EMAB), Loisir et société, 23, 477-508.

Clary E. G., Snyder, M. et Stukas A. A. (1996), Volunteers' motivations: findings from a national survey, Nonprofit and Voluntary Sector Quarterly, 25, 4, 485-505.

Deci E.L. et Ryan R.M. (1985), The general causality orientations scale: selfdetermination in personality, Journal of research in personality, 19, 2, 109-134.

Erikson E.H. (1959), Identity and the life cycle: Selected papers, Psychological Issues, $1,50-100$.

Fennis B.M., Janssen L. et Vohs K.D. (2009), Acts of benevolence: A limited-resource account of compliance with charitable requests, Journal of Consumer Research, 35, 906-924 
Guiot D. (2006), Un cadre d'analyse du processus de vieillissement et de son influence sur le comportement d'achat du consommateur âgé, Recherche et Applications en Marketing, 27, 1, 57-79.

Guiot D. et Urien B. (2012), Comprendre le consommateur âgé : Nouveaux enjeux et perspectives, Première éd., De Boeck.

McAdams D.P. et de St. Aubin E. (1992), A theory of generativity and its assessment through self-report, behavioral acts, and narrative themes in autobiography, Journal of Personality and Social Psychology, 62, 1003-1015.

McAdams D.P., de St Aubin E. et Logan R. (1993), Generativity among young, midlife, and older adults, Psychology and Aging, 8, 221-230.

Pietikainen P. et Ihanus J. (2003), On the origins of psychoanalytic psychohistory, History of Psychology, 6, 2, 171-194.

Prouteau L. et Wolff F. (2004), Donner son temps : les bénévoles dans la vie associative, Economie et Statistique, 372, 3-39.

Prouteau L. et Wolff F. (2007), La participation associative et le bénévolat des seniors, Retraite et Société, 1, 50, 157-189.

Sargeant A., Ford J.B. et West D.C. (2006), Perceptual determinants of nonprofit giving behavior, Journal of Business Research, 59, 155-165

Sue R., et Peter, J.-M. (2011), Intérêts d'être bénévole, Rapport de recherche, laboratoire Cerlis/CNRS Université Paris Descartes. http://www.cnrs.fr/inshs/recherche/docs-vie-labos/interet-etre-benevole.pdf

Tang F., Morrow-Howell N. et Hong S. (2009), Inclusion of diverse older populations in volunteering, Nonprofit and Voluntary Sector Quarterly, 38, 5, 810-827.

Urien B. et Kilbourne W. (2011), On the Role of Generativity and Self-Enhancement Values in Eco-Friendly Behavioural Intentions and Environmentally Responsible Consumption Behaviour, Psychology and Marketing, 28, 1, 69-90.

Van Ingen E. et Dekker P. (2011), Changes in the Determinants of Volunteering: Participation and Time Investment Between 1975 and 2005 in the Netherlands, Nonprofit and Voluntary Sector Quarterly, 40, 4, 682-702. 


\section{Annexe A1 : Les échelles de mesure}

Nous présentons ci-dessous les items et dimensions des différentes échelles de mesure retenus après analyses factorielles exploratoires.

\section{Échelle de motivation envers l'action bénévole (EMAB), adaptée de Chantal et Vallerand (2000)}

En général, pourquoi aidez-vous les autres de façon bénévole ?

Motivations Autodéterminées Altruistes (AA) $\alpha=0,77$

Parce que le bénévolat fait partie de ce que je suis en tant que personne.

Parce que mes activités bénévoles correspondent à ma vraie nature.

Parce que le bénévolat est un reflet de qui je suis réellement.

Motivations Non Autodéterminées Altruistes (NAA) $\boldsymbol{\alpha}=\mathbf{0 , 7 7}$

Parce que je me sentirais honteux-se de ne pas aider les autres.

Parce que je me sentirais mal à l'aise si je ne le faisais pas.

Parce que si je ne faisais pas de bénévolat, je ressentirais des remords.

Motivations Autodéterminées Egoïstes (AE) $\alpha=0,76$

Pour le plaisir d'expérimenter des choses nouvelles lorsque je fais du bénévolat.

Parce que faire du bénévolat est un bon moyen d'entrer en contact avec d'autres personnes.

Parce que le bénévolat est un excellent moyen de faire des rencontres.

Motivations Non Autodéterminées Egoïstes (NAE) $\boldsymbol{\alpha}=\mathbf{0 , 6 0}$

Parce que l'expérience que m'offre le bénévolat pourra éventuellement me servir du côté de l'emploi.

Parce que faire du bénévolat me permet d'enrichir mon curriculum vitae.

Parce que cela me permet d'être mieux considéré-e socialement.

\section{Échelle de l'intérêt génératif adaptée d'Urien et Kilbourne (2011)}

Dimension Postérité (GP) $\alpha=0,85$

Je pense que l'on se souviendra de moi longtemps après ma mort.

Les autres diraient que j'ai apporté des contributions uniques à la société.

C'est comme si je ressentais que ce que j'ai apporté (fait) existera après ma mort.

Dimension Transmission (GT) $\boldsymbol{\alpha}=\mathbf{0 , 7 3}$

J'ai des compétences importantes que j'essaie d'enseigner à d'autres.

J'essaie de transmettre la connaissance que j'ai acquise au travers de mes expériences.

J'ai réalisé et créé des choses qui ont eu un impact sur les autres personnes.

Dimension Communion (GC) $\boldsymbol{\alpha}=\mathbf{0 , 4 5}$

Je me suis engagé auprès de nombreux types de personnes, groupes, et activités dans ma vie.

J'ai la responsabilité d'améliorer le voisinage dans lequel je vis.

\section{Échelle de la sagesse adaptée d'Ardelt (2003) (uniquement la dimension}

\section{«Sagesse affective »)}

Sagesse affective (SA) - Items inversés $\alpha=0,66$

Je n'ai pas toujours vraiment pitié des gens quand ils ont des problèmes.

Il m'est souvent arrivé de ne pas réconforter des gens quand ils en avaient besoin.

Je n'aime pas avoir à écouter les malheurs des autres. 